\title{
FACHCONTROLLING
}

\section{Soziale Unternehmen erfolgreich steuern}

\author{
Andreas Hopmann
}

Ohne eine erfolgreiche Leistungserbringung kann kein Unternehmen auf Dauer überleben. Deshalb genügt auch das betriebswirtschaftliche Controlling allein in sozialen Organisationen nicht, da es nur abbildet, ob die finanziellen Aspekte der Leistungserstellung im vorgegebenen Rahmen liegen, Nur ein sorgfältig konzipiertes und verfolgtes Fachcontrolling dient der erfolgreichen Steuerung der Leistungserbringung selbst.

Controlling kann verstanden werden als strukturierte Selbstüberwachung der Organisation zur Steuerungsunterstützung. Notwendig für diese Selbstüberwachung ist die Festlegung von Zielen. Nur wer Ziele ernsthaft verfolgt, kann mit Controlling sinnvoll steuern. Steuerung ist Leitungsaufgabe. Damit ist auch deutlich, dass nicht die Person, die bestimmte Controlling-Prozesse umsetzt - der Controller oder die Controllerin - diese Ziel festlegt, sondern die Leitungsebene der Organisation. Controlling ist nicht vollständig delegierbar. Dennoch ist es notwendig, bei der Bestimmung der Ziele die Mitarbeiterebene einzubeziehen, die die Leistungen erbringt.

Controlling folgt immer einem Regelkreis (vgl. Abbildung) in dem

\section{Ziele festzulegen sind}

2. die Erreichung dieser Ziele überprüft wird (Soll-Ist-Vergleich)

3. bei Abweichungen von den Zielvorgaben Ursachenanalyse betrieben werden muss

4. um schließlich gegebenenfalls Korrekturen an den Prozessen vorzunehmen.

Dieser Regelkreis ist auf unterschiedliche Prozesse in der Organisation anzuwenden. Ausgangsfrage muss sein: Was will ich steuern? Welche Ziele oder Zielgrößen will ich erreichen - inhaltlich, fachlich, wirtschaftlich?

Für die Umsetzung des Regelkreises werden Prozesse der Berichterstellung (Informationssystem) und Prozesse des Transfers der Berichtsinformationen in das Handeln der Organisation (Transfersystem) benötigt (Horváth 2006). Oft scheitern Controllingsysteme daran, dass der Transfer der Controlling-Information (Kennzahlabweichung von der Zielvorgabe) nicht in Aktion (geändertes Verhalten

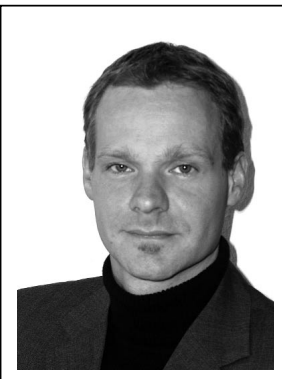

Andreas Hopmann ist Trainer und Organisationsberater in Köln. Im Hauptberuf leitet er das Sachgebiet »Jugendhilfeplanung und Fortbildung « im Landesjugendamt Rheinland. Seine Arbeitsschwerpunkte sind: Entwicklungsprozesse in Organisationen, Planung und Steuerung sozialer Dienstleistungen, Jugendhilfeplanung, Controlling, strategische Planung, Demografie, Szenario-Technik. Internet http://www.hopmanns.de E-Mailconsult@hopmanns.de

der Mitarbeiterinnen und Mitarbeiter) umgesetzt wird. Dieser Transfer ist die große Herausforderung für jedes Controlling-Verfahren. Für ein fachliches Controlling gilt das in besondere Weise, weil hier die Prozesse der Leistungserbringung im Fokus stehen.

\section{Finanzcontrolling vs. Fachcontrolling}

Als in den 1990er Jahren das »Neue Steuerungsmodell« der Kommunalen Gemeinschaftsstelle für Verwaltungsmanagement (KGSt) entwickelt wurde, bestand ein zen-

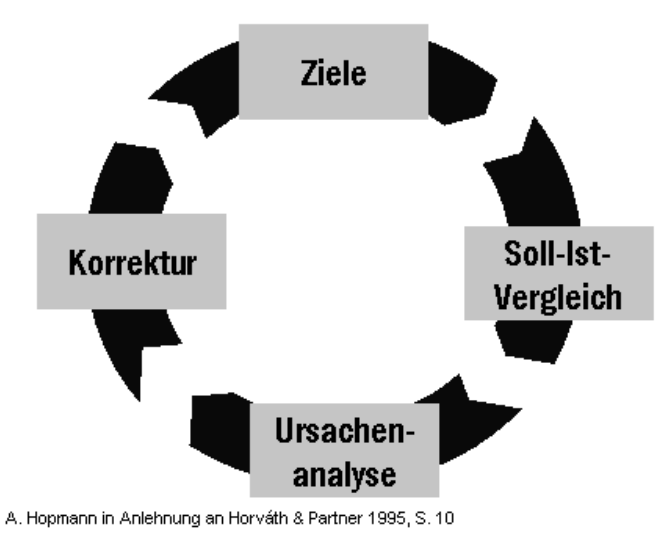

Controlling folgt einem Regelkreis von Zielfestlegung, Überprüfung, Fehlersuche und Korrektur. 
traler Aspekt des Modells darin, Steuerungsinstrumente aus der Betriebswirtschaft in die kommunale Verwaltung zu übertragen (KGSt 1993).

Nun sind Verwaltungen zwar immer wirtschaftende Organisationen gewesen und geblieben, allerdings operieren sie ganz überwiegend nicht wie Unternehmen und können und sollen dieses auch nicht. Ihre Refinanzierungsstruktur beruht nur zum Teil auf leistungsbezogenen Erträgen (z. B. Gebühren) und ihre Leistungsverpflichtungen ergeben sich nicht überwiegend aus Markt- oder individuellen Bürgerbedürfnissen, sondern vor allem aus gesetzlichen Aufträgen. Daher funktionieren bestimmte Instrumente, die auf eine Unternehmenslogik mit Gewinnerzielung ausgerichtet sind, nicht ohne weiteres. Für das Controlling führte man im »Neuen Steuerungsmodell« die Trennung von Finanzcontrolling und Fachcontrolling ein (vgl. u. a. KGSt 1994). Diese Trennung ermöglichte einerseits Finanzaspekte in der Verwaltung abzubilden, die nicht der betriebswirtschaftlichen Logik folgen, anderseits aber »Controlling « in der Form des Fachcontrollings als flächendeckendes Instrument für die Verwaltung anzubieten.

Diese Trennung erscheint auf den ersten Blick künstlich und führt auch bis heute zu Missverständnissen über das, was Controlling in Verwaltungen und in Non-Profit-Organisationen leisten kann und leisten soll. Genau betrachtet ist für diese Organisationen - aber auch für Unternehmen - das Fachcontrolling die zentrale Betrachtungsperspektive. Während das betriebswirtschaftliche Controlling und das Finanzcontrolling nur abbilden können, ob die finanziellen Aspekte der Leistungserstellung im vorgegebenen Rahmen liegen, bildet das Fachcontrolling die Steuerung der Leistungserbringung selbst ab.

Ohne eine erfolgreiche Leistungserbringung hat jede Organisation Schwierigkeiten: Auch eine Bank, die ein Renditeziel von 25 Prozent auf das Eigenkapital anstrebt, wird den Grad der Erreichung diese Zieles im Finanzcontrolling ständig überwachen. Die Abbildung dieser Kennzahl (Eigenkapitalrendite) spiegelt aber letztendlich den Erfolg der Leistungserbringung (in diesem Fall des Bankgeschäftes). Ohne eine Steuerung dieser Leistungserbringung, im Sinne eines Fachcontrollings, sind die Ziele im Finanzcontrolling nicht zu erreichen. Daraus ergibt sich, dass Controlling immer eine finanzielle und eine auf die Leistungserbringung bezogene Komponente benötigt.

\section{Was Fachcontrolling leisten soll}

Fachcontrolling soll dazu beitragen, die notwendigen und geeigneten Leistungen zu angemessenen Kosten anzubieten - wobei sich im Wort "geeignet « auch die Anforderung an die fachliche Qualität verbirgt.

Das Fachcontrolling bezieht sich also auf die Prozesse der Leistungserbringung. Es unterstützt die Umsetzung zentraler auf die Leistungserbringung bezogene Ziele. Dazu gehört auch die Berücksichtigung der eingesetzten Ressourcen.

Für die Jugendhilfe beispielsweise sind das Leistungen, die sich an Kinder, Jugendliche und Familien richten. Daraus ergibt sich ein sehr komplexes Leistungsgesche- hen, das mit vielschichtigen Zielen verbunden ist. Für das Fachcontrolling sind die zentralen Ziele herauszuarbeiten und zu bemessen. Primärer Fokus ist dabei in der Regel die Erzielung positiver Wirkungen für die Zielgruppen. (Hier soll die Frage ausgeklammert werden, inwieweit die Zielgruppen die Sicht der Leistungserbringer und ihrer Auftraggeber dazu im einzelnen teilen. Daneben stehen aus Sicht der Organisation immer auch weitere steuerungsrelevante Ziele, die sich beispielsweise auf weltanschauliche Aspekte, die Wirtschaftlichkeit, den Erhalt der Organisation und vieles mehr beziehen.)

Für das Fachcontrolling ist es notwendig, die steuernden Ziele in einem überschaubaren Rahmen zu halten. Ein unübersichtliches Zielbündel voller gut gemeinter Wünsche wird nicht zu einer zielgerichteten Steuerung beitragen. Viele »Leitbilder « stellen so eine überfordernde Wunsch-Agenda dar, die dann letztendlich oft überhaupt keine Wirkung in der jeweiligen Organisation entwickelt.

\section{Wie man eine Leistungsbeschreibung entwickelt}

Es sind also für jede Leistung wenige tatsächlich steuerungsrelevante Ziele und Zielgrößen festzulegen, die die fachlichen Ziele der Leistungserbringung (Beispiel: erfolgreiche Vermittlung junger Menschen in qualifizierte Ausbildungsverhältnisse) und die Steuerungsziele der Organisation (Beispiel: Personaleinsparung wegen knapper pauschaler Maßnahmenvergütung durch den Kostenträger) in Einklang bringen. Wenn diese realistische Zielfindung vollzogen wurde, kann Fachcontrolling zum Erfolg der Organisation beitragen.

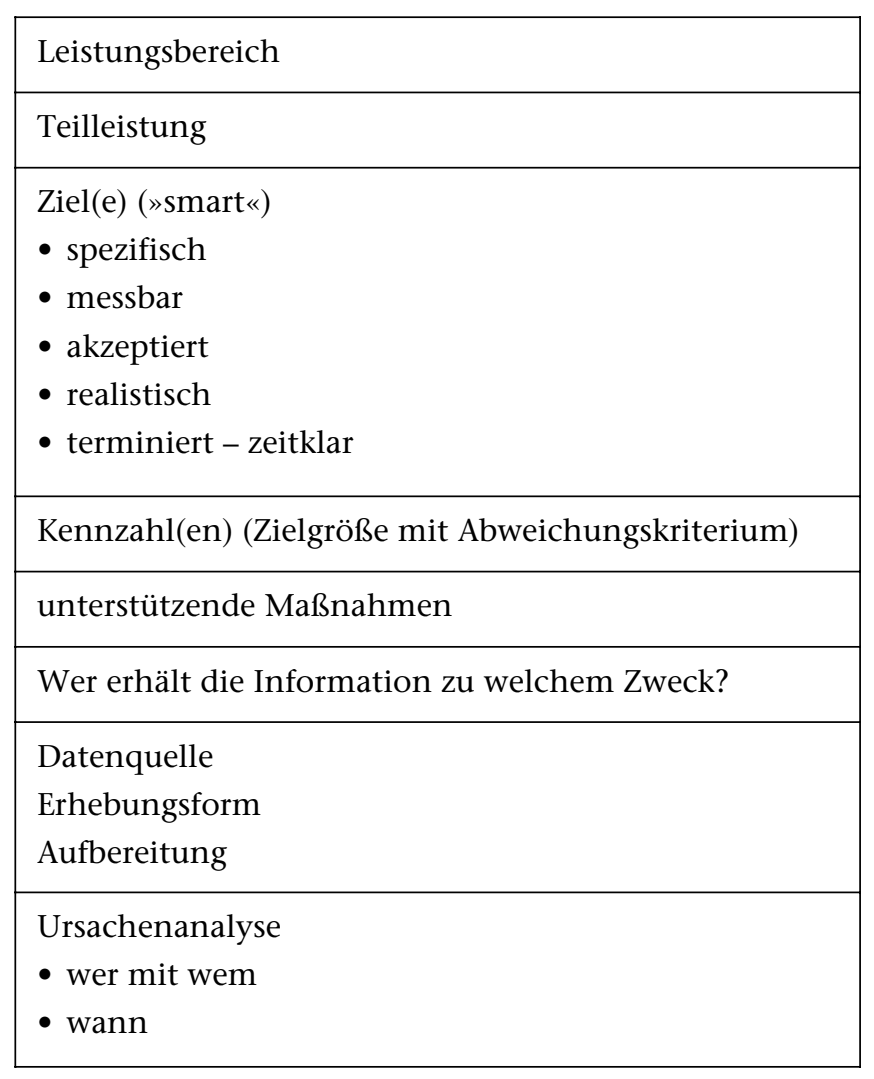

Die Leistungsbeschreibung ist die Grundlage eines funktionierenden Fachcontrollings. 
Notwendig ist eine Leistungsbeschreibung (siehe Tabelle), die eine abgrenzbare Teilleistung mit konkreten Zielen verbindet, auf die sich eine messbare Zielgröße mit einem entsprechenden Abweichungskriterium bezieht. Enthalten sein sollte auch die Formulierung gegebenenfalls notwendiger unterstützender Maßnahmen zur Zielerreichung und das vereinbarte Transferverfahren. Umsetzen lässt sich ein Fachcontrolling dann in drei Schritten:

\section{Erster Schritt: Leistungsbereiche eingrenzen}

Die Einführung von - ernst gemeinten - Controlling-Prozessen stellt Anforderungen an Mitarbeiterinnen und Mitarbeiter, aber auch an die verantwortlichen Leitungskräfte, die die Umsetzung dieser Prozesse fördern und einfordern müssen. Daher ist es oft sinnvoll sich zunächst auf besonders steuerungsrelevante Leistungen und Leistungsbereiche zu konzentrieren. Das kann ein besonders kostenintensiver oder umsatzstarker Bereich sein, das kann aber auch ein qualitativer oder quantitativer Wachstumsbereich sein, in dem hohe Chancen oder Risiken liegen.

Beispiel: Ein Träger der freien Wohlfahrtspflege, eingetragener Verein unter dem Dach eines großen Wohlfahrtsverbandes, bietet unter anderem betreutes Wohnen für junge Menschen an. Diese Leistung wird in der Regel zu 100 Prozent von den belegenden Jugendämtern bezahlt.

Die Einrichtung diese Angebotes in angemieteten Wohnungen mit Betreuung durch die sozialpädagogischen Fachkräfte des Trägers war vor zwei Jahren als zusätzliches Wachstumsstandbein initiiert worden. Der wirtschaftliche und fachliche Erfolg bleibt bisher aus, da keine ausreichenden Belegungszahlen erreicht werden.

Als ein zentrales Problem werden häufige Maßnahmeabbrüche in der Starphase der Einzelfallhilfen identifiziert. Diese erfordern einen hohen Personalaufwand in der Eingangsphase, führen zu einer Zurückhaltung der Jugendämter bei der Belegung - der Ruf leidet - und dokumentieren nicht zuletzt die Unzufriedenheit der betreuten jungen Menschen.

\section{Zweiter Schritt: Ziele formulieren und Zielgrößen festlegen}

Die bewusste Auseinandersetzung mit Zielen führt zu mehr Klarheit über die eigenen Interessen und Steuerungsnotwendigkeiten. Diese lassen sich in der Regel nicht in einer einfachen gemeinsamen Kennzahl abbilden. Das gilt nicht nur in der Jugendhilfe: Erfolgreiche Bankprodukte zu entwickeln, am Markt nachgefragte Autos zu bauen und gute erzieherische Hilfen zu erbringen ist nicht über eine oder wenige Kennzahlen abbildbar.

Fachcontrolling unterstützt auch die konsequente Umsetzung von Zielen, die Mitarbeiterinnen und Mitarbeiter als »selbstverständlich « ansehen. Ob aber tatsächlich die- se »Selbstverständlichkeiten « Bestandteil des täglichen fachlichen Handelns sind, wird oft erst durch strukturierte Controlling-Prozesse sichtbar. Es ist also zu formulieren: Was sind unsere primären Ziele in Bezug auf die konkrete Leistung? Dabei sind die möglichen

Ziel-Dimensionen vielfältig, u. a.:

- fachliche Erfolgskriterien

- Wirtschaftlichkeit

- Auslastung

- Marktführerschaft (Qualität: beste oder innovativste Leistung, Quantität: größter Anbieter)

- Preisführerschaft (billigster Anbieter oder PreisLeistungs-Sieger)

- Kundenzufriedenheit

- Zufriedenheit der Auftraggeber

- Zufriedenheit der Klienten

Die Ziele »smart « - spezifisch , messbar, akzeptiert, realistisch, terminiert - zu formulieren ist eine gute Hilfestel-

\section{»Das Fachcontrolling muss für soziale Organisation die zentrale Betrachtungsperspektive sein «}

lung in der Praxis. Um einseitige Fokussierungen zu vermeiden bietet es sich an, mehrere Ziele (maximal drei) und Kennzahlen pro Teilleistung zu bilden. Ziele sollten zunächst für einen angemessenen aber überschaubaren Zeitraum festgelegt werden (bis zu einem Jahr).

Darüber hinaus ist sehr wichtig festzulegen, ob und welche unterstützenden Maßnahmen notwendig sind, um das vorgegebene Ziel zu erreichen. Ein einfaches Verschieben der Messlatte weist nicht den Weg, wie diese »übersprungen« werden kann.

Für unser Beispiel könnte als Zielvorgabe für die Leistung »betreutes Wohnen « eine durchschnittliche Auslastung der vorhandenen Plätze zu 65 Prozent (bisher unter $50 \%)$ als Mindestgröße vorgegeben werden.

Der Anteil der innerhalb acht Wochen vorzeitig beendeten Maßnahmen soll auf 15 Prozent begrenzt werden (derzeit bei geringen Fallzahlen ca. 33\%).

Als unterstützende Maßnahmen werden folgende Schritte vereinbart:

- sorgfältigere Prüfung der von den Jugendämtern zur Betreuung vorgeschlagenen jungen Menschen, intensive Hilfeplanung mit den betroffene Jugendlichen und dem jeweiligen Jugendamt (verbunden mit dem Verzicht auf einzelne Betreuungsmaßnahmen). 
- leicht erhöhte Personalstunden für die »Eingewöhnungsphase «

- Kündigung einer der drei angemieteten Wohnungen (»Qualität vor Quantität«)

\section{Dritter Schritt: Soll-Ist-Vergleich und Transfer-Verfahren}

Es müssen klare Verantwortlichkeiten formuliert werden, wann die Zielerreichung erstmalig überprüft wird. Damit sollte nicht bis zum Ablauf des vereinbarten Steuerungsintervalls abgewartet werden, da dann bei gravierenden Abweichungen jede Steuerung erst sehr spät erfolgen kann. Andrerseits muss dieser erste Berichtszeitraum so gewählt sein, dass auch Veränderungen darstellbar sind. Mindestzeitraum sollten drei Monate sein.

Im Transferverfahren muss vereinbart sein, wer wann mit wem die Ursachenanalyse bei einer Zielabweichung durchführt. Das sollte die unterste Leitungsebene gemeinsam mit den für die Leistungserbringung verantwortlichen Mitarbeiterinnen und Mitarbeitern sein. Als letzte Stufe muss klar sein, bis wann muss eine neue Zielvereinbarung auf Grundlage dieser Analyse vorliegen muss.

Nach drei Monaten ist in unserem Beispiel zum betreuten Wohnen ein deutlicher Rückgang der Abbruchquoten feststellbar (unter $20 \%$ ). Bei der Ursachenanalyse wird deutlich, dass die sorgfältige Auswahl der jungen Menschen dazu stärker beiträgt als der - teure - erhöhte Personaleinsatz in der Eingewöhnungsphase. Zunächst soll der Personaleinsatz aber bleiben. Die Auslastung ist allerdings nur relativ - durch die Reduzierung der verfügbaren Plätze - verbessert worden. Es wird ergänzend vereinbart, dass die Geschäftsleitung verstärkt persönliche Kontakte zu den belegenden Jugendämtern aufnimmt, um auf die Änderungen in der Aufnahmepraxis und die damit verbundenen Erfolge hinzuweisen.

Es wird deutlich, dass eine enge Einbindung der Handlungsebene notwendig ist. Die Mitarbeiterinnen und Mitarbeiter generieren durch ihr Handeln die Arbeitsweise der jeweiligen Organisation. Wenn an dieser Arbeitsweise etwas anders - besser - werden soll, kann das nur durch Verhaltensänderungen auf dieser Ebene gelingen.

\section{Literatur}

Hopmann, Andreas: Controlling und Steuerung. Elemente oder neues Verständnis von Planung? In: Maykus, Stephan (Hg.): Herausforderung Jugendhilfeplanung, Weinheim und München 2006.

Horváth \& Partners: Das Controllingkonzept, 6. Aufl., München 2006.

KGSt: Das neue Steuerungsmodell. KGSt-Bericht 05/1993, Köln 1993.

KGSt: Verwaltungscontrolling im Neuen Steuerungsmodell. KGSt-Bericht 15/1994, Köln 1994.
Eine besondere Rolle spielt im Fachcontrolling die untere Leitungsebene. Hier liegen die Schlüsselpositionen für die Handlungssteuerung der Organisation. Diese Leitungskräfte (Teamleitungen, Abteilungsleitungen) müssen den vollständigen Ablauf des Controlling-Regelkreises inklusive der Transferschritte gewährleisten. Sie müssen überprüfen, ob tatsächlich Verhaltensänderungen bei den Mitarbeiterinnen und Mitarbeitern stattfinden und diese gegebenenfalls einfordern.

\section{Resümee}

Fachcontrolling dient der Steuerung der Leistungserbringung einer Organisation über das Handeln ihrer Mitarbeiterinnen und Mitarbeiter. Damit führt es zu fachlichem, ideellem und auch wirtschaftlichen Erfolg. Controlling bedeutet also nicht Kennzahlen zu entwickeln und Berichte zu schreiben, sondern Verhalten zu ändern.

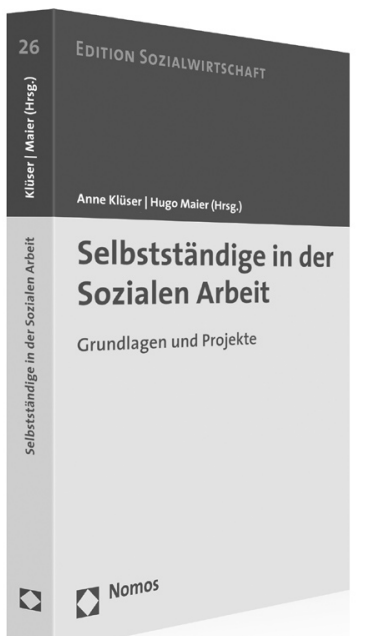

\section{Selbstständige in der Sozialen Arbeit Grundlagen und Projekte \\ Herausgegeben von Dr. Anne Klüser und Prof. Dr. Hugo Maier \\ 2009, 263 S., brosch., 44,- €, ISBN 978-3-8329-4111-6 \\ (Edition Sozialwirtschaft, $B d .26)$}

Berufliche Selbstständigkeit in der Sozialen Arbeit: Heute ist kaum noch ein Bereich denkbar, in dem dies nicht möglich wäre. In vielen Feldern Sozialer Arbeit sind Selbstständige inzwischen etabliert und zu einer festen Größe avanciert. Sie setzen neue Standards, fordern die traditionellen Wohlfahrtsverbände heraus und bringen so Bewegung in die Soziale Arbeit.

\section{Nomos}

Bitte bestellen Sie im Buchhandel oder versandkostenfrei unter $\downarrow$ www.nomos-shop.de 